\title{
The degree of microbiome complexity influences the epithelial response to infection Jeffrey J Mans ${ }^{\dagger 1}$, Kate von Lackum ${ }^{\dagger 1}$, Cassandra Dorsey ${ }^{1}$, Shaun Willis ${ }^{1}$, Shannon M Wallet ${ }^{1,2}$, Henry V Baker ${ }^{3}$, Richard J Lamont ${ }^{1}$ and Martin Handfield*1
}

Address: ${ }^{1}$ Department of Oral Biology, College of Dentistry, University of Florida, Gainesville, Florida, ${ }^{2}$ Department of Periodontology, College of Dentistry, University of Florida, Gainesville, Florida and ${ }^{3}$ Department of Molecular Genetics and Microbiology, College of Medicine, University of Florida, Gainesville, Florida

Email: Jeffrey J Mans - jmans@dental.ufl.edu; Kate von Lackum - kvonlackum@dental.ufl.edu; Cassandra Dorsey - cdorsey@dental.ufl.edu; Shaun Willis - swillis@dental.ufl.edu; Shannon M Wallet - swallet@dental.ufl.edu; Henry V Baker - hvbaker@ufl.edu;

Richard J Lamont - rlamont@dental.ufl.edu; Martin Handfield* - mhandfield@dental.ufl.edu

* Corresponding author †Equal contributors

Published: 18 August 2009

BMC Genomics 2009, 10:380 doi:10.1 186/147|-2164-10-380
Received: 13 November 2008

Accepted: 18 August 2009

This article is available from: http://www.biomedcentral.com/I47I-2/64/I0/380

(C) 2009 Mans et al; licensee BioMed Central Ltd.

This is an Open Access article distributed under the terms of the Creative Commons Attribution License (http://creativecommons.org/licenses/by/2.0), which permits unrestricted use, distribution, and reproduction in any medium, provided the original work is properly cited.

\begin{abstract}
Background: The human microflora is known to be extremely complex, yet most pathogenesis research is conducted in mono-species models of infection. Consequently, it remains unclear whether the level of complexity of a host's indigenous flora can affect the virulence potential of pathogenic species. Furthermore, it remains unclear whether the colonization by commensal species affects a host cell's response to pathogenic species beyond the direct physical saturation of surface receptors, the sequestration of nutrients, the modulation of the physico-chemical environment in the oral cavity, or the production of bacteriocins. Using oral epithelial cells as a model, we hypothesized that the virulence of pathogenic species may vary depending on the complexity of the flora that interacts with host cells.

Results: This is the first report that determines the global epithelial transcriptional response to co-culture with defined complex microbiota. In our model, human immortalized gingival keratinocytes (HIGK) were infected with mono- and mixed cultures of commensal and pathogenic species. The global transcriptional response of infected cells was validated and confirmed phenotypically. In our model, commensal species were able to modulate the expression of host genes with a broad diversity of physiological functions and antagonize the effect of pathogenic species at the cellular level. Unexpectedly, the inhibitory effect of commensal species was not correlated with its ability to inhibit adhesion or invasion by pathogenic species.

Conclusion: Studying the global transcriptome of epithelial cells to single and complex microbial challenges offers clues towards a better understanding of how bacteria-bacteria interactions and bacteriahost interactions impact the overall host response. This work provides evidence that the degree of complexity of a mixed microbiota does influence the transcriptional response to infection of host epithelial cells, and challenges the current dogma regarding the potential versus the actual pathogenicity of bacterial species. These findings support the concept that members of the commensal oral flora have evolved cellular mechanisms that directly modulate the host cell's response to pathogenic species and dampen their relative pathogenicity.
\end{abstract}




\section{Background}

The human microflora is an extremely complex ecosystem characterized by the simultaneous presence of a large number of "normal" colonizers, associated with health, and thriving in a dynamic environment alongside opportunistic and pathogenic species. Since health is the most common state of a host, it has been speculated that the autochthonous flora has co-evolved with its host to interact in a balanced state that is beneficial to both the host and the microflora. There are an appreciable number of benefits to the host that the indigenous flora is thought to provide, including the synthesis of vitamins (B complex and $\mathrm{K}$ ), the prevention of infection by pathogens (by direct competition for niches or by immune cross-reactivity), and its impact in the normal development of the immune system [1,2]. Indeed, observed differences between pathogen-stimulated and commensal-stimulated immune responses are suggested to provide important insights toward understanding the molecular aspects of host-microbiota interactions [2-4]. Most notable examples include the intestinal commensals whose interactions with the gut epithelium trigger both innate and adaptive immune responses, influence epithelial cell proliferation [5], and induce commensal-specific IgA production and secretion in the gut to keep commensal species in check [6]. More recently, an oral commensal organism, Streptococcus salivarius strain K12, was demonstrated to antagonize Pseudomonas aeruginosa-induced IL-8 secretion from human bronchial epithelial cells, suggesting a role for commensal species in modulating human epithelial cell immune responses in the nasopharynx [4]. Likewise, a separate group has described the capability of $S$. cristatus and certain other streptococcal species to dampen the IL8 response induced by infection with the periodontal pathogen Fusobacterium nucleatum in four different epithelial cell lines. These observations demonstrate that polymicrobial infection of epithelial cells with oral streptococcal species and commonly associating pathogens can attenuate the proinflammatory effects elicited during infection of these cells with the pathogens [7].

Since host and microbiota interactions are inherently unstable, disease may arise at the mucosal surface of a susceptible host when a perturbation occurs in the epithelial environment leading to "unintended" (in an evolutionary sense) consequences of immune or other host cell activity. Such instances include both hyper-acute immune responses as well as the converse situation, such as when the host becomes immuno-compromised. Further, the complex etiology of oral infectious diseases involves consortia of bacteria thriving in biofilms and working in concert with immunological susceptibilities in the host. In particular, periodontal diseases are a group of infections that lead to damage of the periodontium and ultimately, exfoliation of the teeth, and these infections are among the most common bacterial diseases of humans $[8,9]$. As complex as these multifactorial inflammatory diseases are, there is a consistent relationship between the Gramnegative anaerobe Porphyromonas gingivalis and severe, chronic manifestations of the disease [8-11]. However, while bacteria have traditionally been viewed as strictly beneficial or harmful, it is our contention that these oversimplified designations are no longer appropriate, and that expression of an organism's potential pathogenicity is context dependent [12]. The current genomic revolution offers an unprecedented opportunity to identify the molecular foundations of host-commensal and hostpathogen relationships so that we can understand how they assist or interfere with our normal physiology [2]. In line with a broader contextual view of relative potential pathogenicity, transcriptional profiling has emerged as a tool that allows the host to report the level of disruption induced by bacteria in the absence of preconceived notions regarding bacterial "intentions" (reviewed in $[12,13])$.

The initial interface between periodontopathic organisms, such as $P$. gingivalis, or members of the normal flora such a Streptococcus gordonii and the host is the epithelial layer in the subgingival crevice. Epithelial cells have traditionally been considered a passive barrier to infection. There is, however, growing evidence that they contribute more significantly to host defense than previously appreciated. There is a significant body of literature that reports that these cells efficiently signal a microbial intrusion to the immune cells to ensure effective mobilization of the innate and specific defense mechanisms. Furthermore epithelial cells can produce oxidants and antimicrobial peptides to actively participate in fending off intruding microbes [14,15]. Consequently, several organisms have evolved to circumvent the specific defenses available to the epithelium. For example, $P$. gingivalis invades epithelial cells and remains viable intracellularly in a rather covert manner whereby host cell programmed cell death is suppressed [16]. P. gingivalis stimulates integrin-dependent signaling in host cells to effect invasion and subsequently resides in the perinuclear area in epithelial cells [17]. In fact, besides $P$. gingivalis, an intracellular location has been suggested to be a natural component of the lifestyle of a number of other oral organisms [18-20]. Consequently, the regulation of normal processes such as cell division or apoptosis may be key events to a balanced longstanding intracellular state whereby microbes of the oral cavity and host cells co-exist and inflict a minimal degree of harm on each other.

In regards to the extracellular source for this pathogen, numerous studies demonstrate that $P$. gingivalis aggregates with common members of supragingival plaque including the periodontal pathogen Aggregatibacter actinomyce- 
temcomitans, commonly associated with Localized Aggressive Periodontitis (LAP), and numerous oral streptococci ubiquitous to early plaque biofilms [21-23]. Interestingly, $P$. gingivalis only forms biofilms with $S$. gordonii and other oral streptococci but not $S$. mutans species $[24,25]$, the specific molecular adherence strategies of which are well documented [24,26-29]. Recently, it was discovered that approximately $1 \%$ to $2 \%$ of the $P$. gingivalis genome was regulated during the initial stages of development of a community with $S$. gordonii and contribute to the development of heterotypic biofilms [26]. Pioneering organisms and their interspecies interactions thus ultimately determine the diversity potential of heterotrophic biofilms developing on newly acquired tooth pellicle. One cannot consider the pathogenicity of single periodontal organisms without considering the biofilm context that brought them to the host interface. The encounter between host and microbiota may thus be a finely tuned set of interactions whereby both cell types co-exist with each other. Hence, the transcriptional status of gingival cells has significance for the specific response to individual organisms and to overall well-being of the host (reviewed in $[12,13]$ ).

This is the first report that determines the epithelial transcriptional response to co-culture with complex microbiota constituted with $P$. gingivalis and $S$. gordonii, two interacting co-inhabitants of the oral cavity. Colonization of the oral cavity with $S$. gordonii and related streptococci is one of the first steps in the development of plaque upon the acquired pellicle of a recently cleaned tooth structure. $P$. gingivalis, a later colonizing oral inhabitant, is associated with chronic gingival disease and can result in severe periodontal tissue destruction. The host response to oral colonization has both pathophysiological and temporal effects on disease progression induced by late colonizing pathogenic species. It is often the balance between commensal, opportunistic, and pathogenic species in a particular microbiota that can tip the scales in favor of periodontal health or disease [30]. Studying the patterns of gene expression induced by co-infection with a complex microbiota can help reflect a more biologically and physiologically relevant host-pathogen interplay. We investigated whether the presence of an oral commensal in co-culture with gingival epithelial cells and $P$. gingivalis in a mixed microbial challenge would impact the epithelial response. The data presented herein supported the concept that $S$. gordonii does impart profound and global transcriptional changes in the epithelial response to a $P$. gingivalis challenge. In addition, colonization with commensal species directly modulated the host cell's response to co-infection with a pathogenic species and antagonized the ability of $P$. gingivalis to modulate cell cycle, yet had little effect of the invasiveness of this pathogenic species.

\section{Results \\ Impact of Co-Culture on Adhesion and Invasion Levels of Bacteria to HIGK}

Many studies have shown that $S$. gordonii and $P$. gingivalis demonstrate vastly different interaction characteristics with gingival epithelial cells. The former aggregates on the outside of epithelial cells and the latter invades within twenty minutes of infection, and resides in a perinuclear localization [31-33]. The interaction characteristics of $P$. gingivalis with HIGK in mono- and mixed cultures were first investigated to establish the baseline of this system. Consistent with previous reports, at a Multiplicity of Infection (MOI) of 2500:1 and in mono-infection, a great majority of the $S$. gordonii remained extracellular and interacted to a total level of $20 \pm 1$ colony forming units (CFU) per HIGK cell. In contrast, $P$. gingivalis was recovered mostly intracellularly under the conditions used in this study, and at a level of approximately $25 \pm 1$ CFU per HIGK cell. To determine whether the level of invasion by $P$. gingivalis was impacted by co-culture in the presence of commensal species, antibiotic protection assays were conducted on cells co-infected with $P$. gingivalis and $S$. gordonii using the same infection parameters as in the monospecies infection experiments. Although invasion levels of $P$. gingivalis were reduced by co-infection with $S$. gordonii, the effect was not statistically significant. Similarly, the levels of adhesion of $S$. gordonii were not significantly impacted by co-culture with $P$. gingivalis (data not shown). Under the experimental conditions used herein, the HIGK monolayer remained of a normal appearance and morphology and did not appear to be affected by the co-culture with either of the bacterial species at $2 \mathrm{~h}$ postinfection. Microscopic observations supported the finding that co-culture with a complex flora, neither abrogated nor potentiated the invasiveness of $P$. gingivalis (data not shown). Hence, bacteria-bacteria interactions that occur in this complex flora model and under the conditions used herein did not significantly impact the invasive phenotype of $P$. gingivalis.

\section{A Complex Infection Elicits a Different Epithelial Transcriptome}

Assessment of the HIGK transcriptional response during interaction with $P$. gingivalis, $S$. gordonii, or a mixture of the two organisms was performed to determine the global impact of a mixed microbial challenge on epithelial cells. Signal intensity data for the probe sets were categorized by unsupervised analysis using Cluster [34] and supervised class prediction with BRB Array Tools [35]. Relative intensity values were displayed using Treeview [34]. The unsupervised hierarchical clustering analysis showed that the biological replicates clustered together (data not shown). This demonstrated that each treatment condition generated a unique transcriptional response in HIGK that was 
consistent and reproducible amongst biological replicates. Supervised class prediction utilizing a combination of several prediction methods and a random variance model for univariate $\mathrm{F}$ tests [35] revealed 6066 probe sets were differentially expressed among the treatment conditions, at a level of significance of $p<0.05$. Figure 1 shows a Treeview representation of the 6066 probe sets differentially expressed amongst the four treatments. A two hour mono-infection with $S$. gordonii and $P$. gingivalis alone demonstrated unique transcriptional responses that were diametrically opposite to one another, as well as distinct when compared to uninfected HIGK. At this early infection time point, the HIGK's transcriptional response to mixed infection appears most similar to the transcriptional pattern of the HIGK infected with S. gordonii alone. These data suggested that, under mixed infection conditions, the commensal species had the ability to inhibit and reprogram the transcriptional response elicited by $P$. gingivalis. The antagonistic effect of $S$. gordonii on the transcriptional response to cells co-infected with $P$. gingivalis could not be ascribed to different invasion levels of $P$. gingivalis in mixed challenges.

While mixed co-cultures of pathogen with commensal species clustered closer to the signatures associated with $S$. gordonii alone, ontology analysis was undertaken to elucidate the biological relevance of the cellular pathways most differentially impacted by a mixed infection condition. The 6066 probe sets that were differentially impacted at a significance level of $p<0.05$ amongst the infection conditions were used to populate known KEGG pathways using Pathway Express software [36-39]. One feature of this software is the use of statistical algorithms to determine the most highly impacted pathways, considering the number of input genes compared to total genes in a given pathway; a commonly used hypergeometric over-representation approach. The most highly impacted pathways $(p<0.05)$ populated by genes differentially expressed (also $p<0.05$ ) are listed in Table 1. As it was the most significantly impacted pathway in this analysis $(p<0.001)$, the cell-cycle pathway was selected for further phenotypic validation. As indicated in Table 1, a total of 45 cell cycleassociated genes were differentially modulated by a complex flora as compared to mono-infection with P. gingivalis. A significant proportion of two key classes of regulatory molecule genes - cyclins and their cognate cyclin-dependent kinases (CDK) - were differentially regulated in HIGK under each infection condition. Cyclins are transiently expressed nuclear proteins that are required for CDK activation that drives progression through a cell cycle [40]. A summary of the major cell cycle regulatory molecules impacted by infection with $P$. gingivalis and $S$. gordonii is presented in Figure 2.

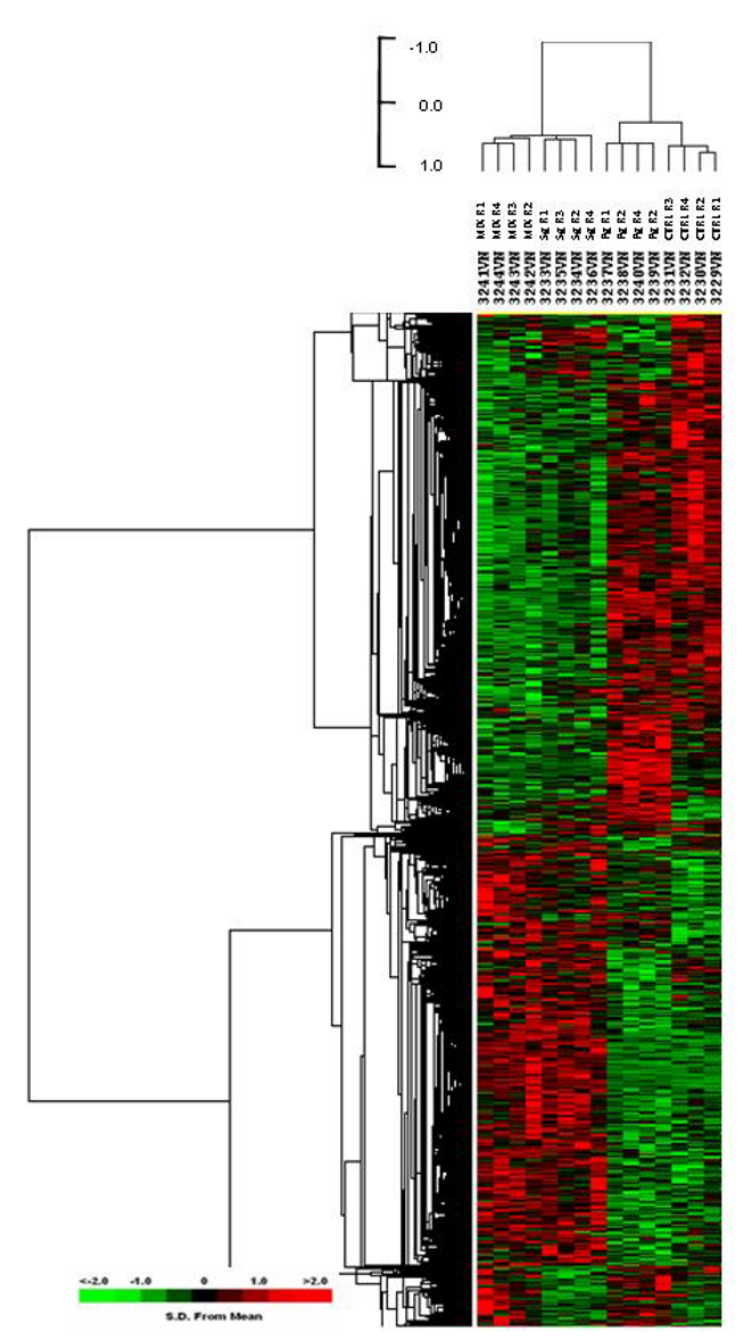

\section{Figure I}

HIGK Gene expression upon $P$. gingivalis and $S$. gordonii single- and mixed infections. Hierarchical clustering was performed on variance-normalized signal of gene expression data from uninfected HIGK cells (CTRL) and from cells in co-culture with either organism or a mixture of both for 2 $\mathrm{h}$ before RNA isolation and purification. Probe set signal intensities were variance-normalized, mean-centered across samples, and subjected to hierarchical cluster analysis. Heat map and dendrogram were constructed from 6066 probe sets that were differentially expressed among the treatment conditions at a level of significance of $p<0.05$. The degree of similarity between the transcriptional profiles of each sample is expressed by Pearson's correlation coefficient distance metric, according to the adjacent scale. The expression state of each data point is represented as standard deviations from the mean expression level for that gene in all samples. Red indicates a relative increase, green indicates a relative decrease, and black indicates no relative change of mRNA transcripts for a given gene. 
Table I: HIGK gene ontology comparing $P$. gingivalis and $S$. gordonii co-infectionto $P$. gingivalis mono-infection. ${ }^{a}$

\begin{tabular}{|c|c|c|c|}
\hline Impacted pathwayb & Impact factorc & \# of modulated genes (total) in Pathwayd & $p$-value \\
\hline Cell cycle & 13.542 & $45(112)$ & $<0.001$ \\
\hline Ubiquitin mediated proteolysis & 11.305 & $22(45)$ & $<0.001$ \\
\hline Adherens junction & 8.788 & $29(77)$ & $<0.001$ \\
\hline TGF-beta signaling pathway & 7.989 & $29(84)$ & 0.002 \\
\hline SNARE interactions in vesicular transport & 7.691 & $16(36)$ & 0.001 \\
\hline Apoptosis & 7.513 & $30(84)$ & 0.002 \\
\hline Jak-STAT signaling pathway & 6.989 & 46 (I53) & 0.003 \\
\hline MAPK signaling pathway & 6.207 & $75(273)$ & 0.009 \\
\hline Wnt signaling pathway & 5.208 & $42(147)$ & 0.02 \\
\hline Colorectal cancer & 5.184 & $23(77)$ & 0.05 \\
\hline Notch signaling pathway & 5.134 & $16(46)$ & 0.03 \\
\hline
\end{tabular}

aThe epithelial cell pathways were determined by Pathway Express.

bKyoto Encyclopedia of Genes and Genomes pathways http://www.genome.jp/kegg/.

cThe impact factor measures the pathways most affected by the changes in gene expression by considering the proportion of differentially regulated genes, the perturbation factors of all the pathway genes, and the propagation of these perturbations throughout the pathway. Only pathways with an impact factor greater than 5 are included.

dNumber of regulated genes in a pathway/total number of genes currently mapped to this pathway.

\section{Effect of a Mixed Flora Challenge on the Epithelial Cell Growth Dynamic}

The finding that cell cycle regulation was significantly impacted in HIGK cells encountering mono- versus mixed infection was confirmed at the cellular level using twocolor fluorescence-activated cell sorting (FACS) cytometric analysis. The percentage of HIGK in any given phase of the cell cycle was analyzed using HIGK co-cultured with $P$. gingivalis and $S$. gordonii in mono- and mixed-cultures in the presence of BrdU (Figure 3). The ratios of BrdU amounts incorporated during DNA replication of $S$ phase, compared to total DNA content and overall cell size, reflects the cell cycle phase of a given cell. A minimum of $1 \times 10^{4}$ cells were analyzed in each condition to determine the proportion of HIGK in different cell cycle phases (Figure 3, panel G). The greatest differences infection status imparted upon HIGK cell cycling were observed during $S$ phase, which was consistent with the mRNA profiling where high proportion of S-phase promoting factor genes (Figure 3) were impacted by infection with a mixture of $S$. gordonii and $P$. gingivalis, compared to mono-infection with pathogen alone. Infection with $S$. gordonii resulted in the majority of HIGK remaining in S phase while infection with $P$. gingivalis resulted in a wider distribution of HIGK in each of the various stages of cell cycling, mirroring uninfected controls. Introduction of the commensal $S$. gordonii to $P$. gingivalis-infected HIGK cells promoted progression of HIGK into S-phase of the cell-cycle, shifting the cycling trend towards that of cells challenged only with the commensal species (Figure 3). What remained consistent amongst the microarray and phenotypic experiments was that HIGK infected with a mixture of $S$. gordonii and P. gingivalis cycled similarly to cells mono-infected with $S$. gordonii.
FACS analysis confirmed microscopic observations and CFU data on $P$. gingivalis interaction characteristics with HIGK. As shown in Figure 3 (blue dots), the level of total interaction was similar $(p=0.106)$ whether $P$. gingivalis was in single (B) or in mixed (D) co-cultures with HIGK. Thus, both the total numbers of $P g$ interacting per cell (Figure 4A), as well as the total numbers of HIGK that interact with at least a single $P g$ bacterium (Figure 3B, D) were both not significantly influenced by $S g$ coinfection compared to mono- $\mathrm{Pg}$ infection. This corroborates the notion that the observed shift in cycling behavior of HIGK co-cultured with $P$. gingivalis and $S$. gordonii compared to that of HIGK infected with $P$. gingivalis alone was not due to the inhibition of $P$. gingivalis adhesion or invasion. Transcriptionally at 2-hours, and phenotypically at $24 \mathrm{~h}$ post-infection, commensal species in a mixed infection can thus influence HIGK's physiological response towards one that is characteristic of HIGK cells mono-infected with a commensal species.

To reconcile the transcriptional snap-shot and the cellcycle analysis into a coherent model, a direct measurement of cell division time was performed over a period of six days after a $2 \mathrm{~h}$ infection. As shown in Figure 4B, mono-infection with $P$. gingivalis stimulated an HIGK growth rate resembling that of uninfected controls $(p>$ $0.05)$. In contrast, mono-infection with $S$. gordonii constrained growth of HIGK as compared to non-infected controls $(p<0.05)$. Mixed infection of HIGK with $S$. gordonii and $P$. gingivalis resulted in a dose-dependant inhibition of cell growth where $S$. gordonii antagonized the growth stimulatory effect of $P$. gingivalis at the lowest MOI tested (100:1), but seen with more significance at higher MOIs (500:1) (2500:1) $(p<0.05)$. The constrained growth observed under $S$. gordonii infection conditions 
A.

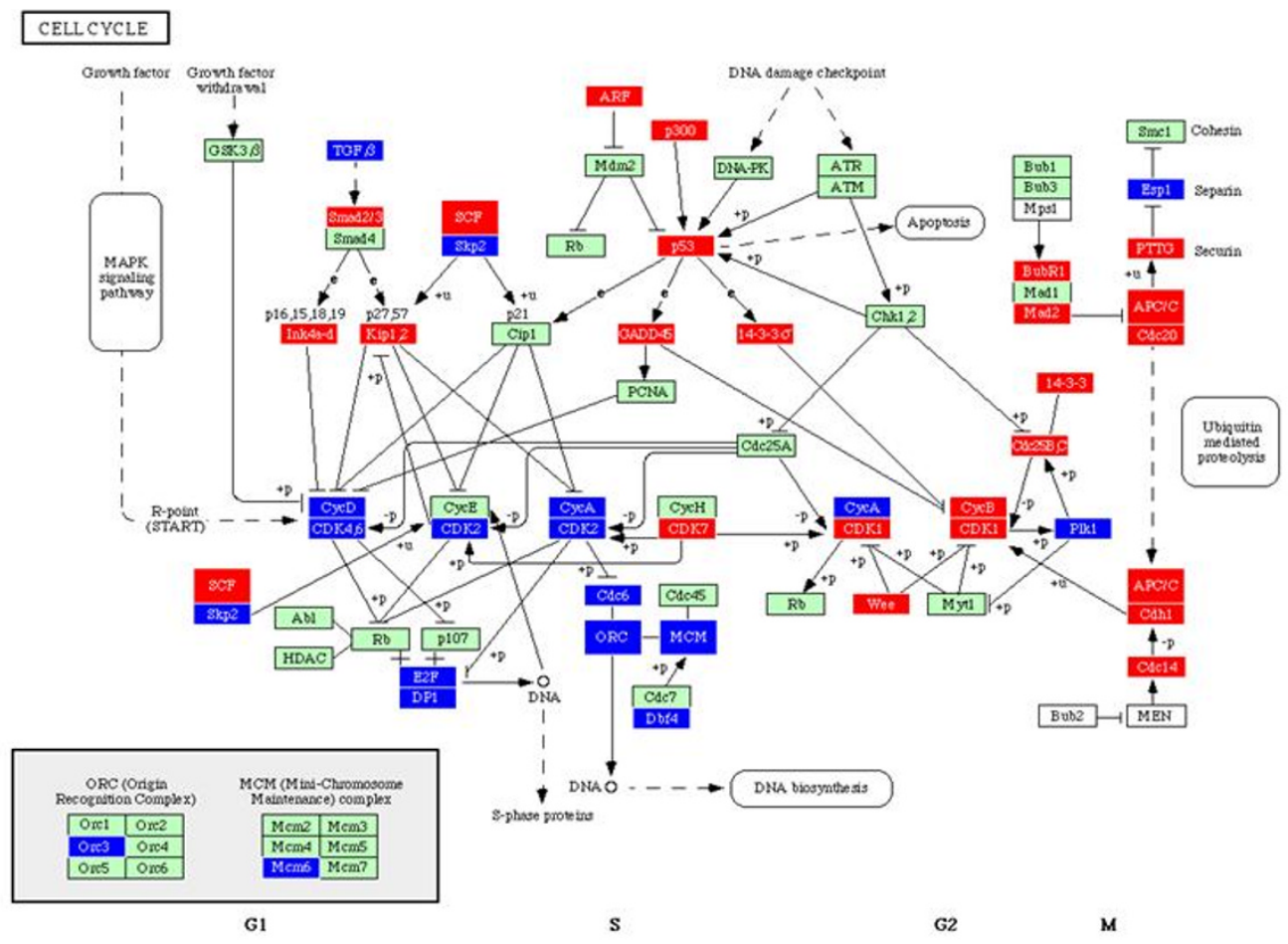

B.

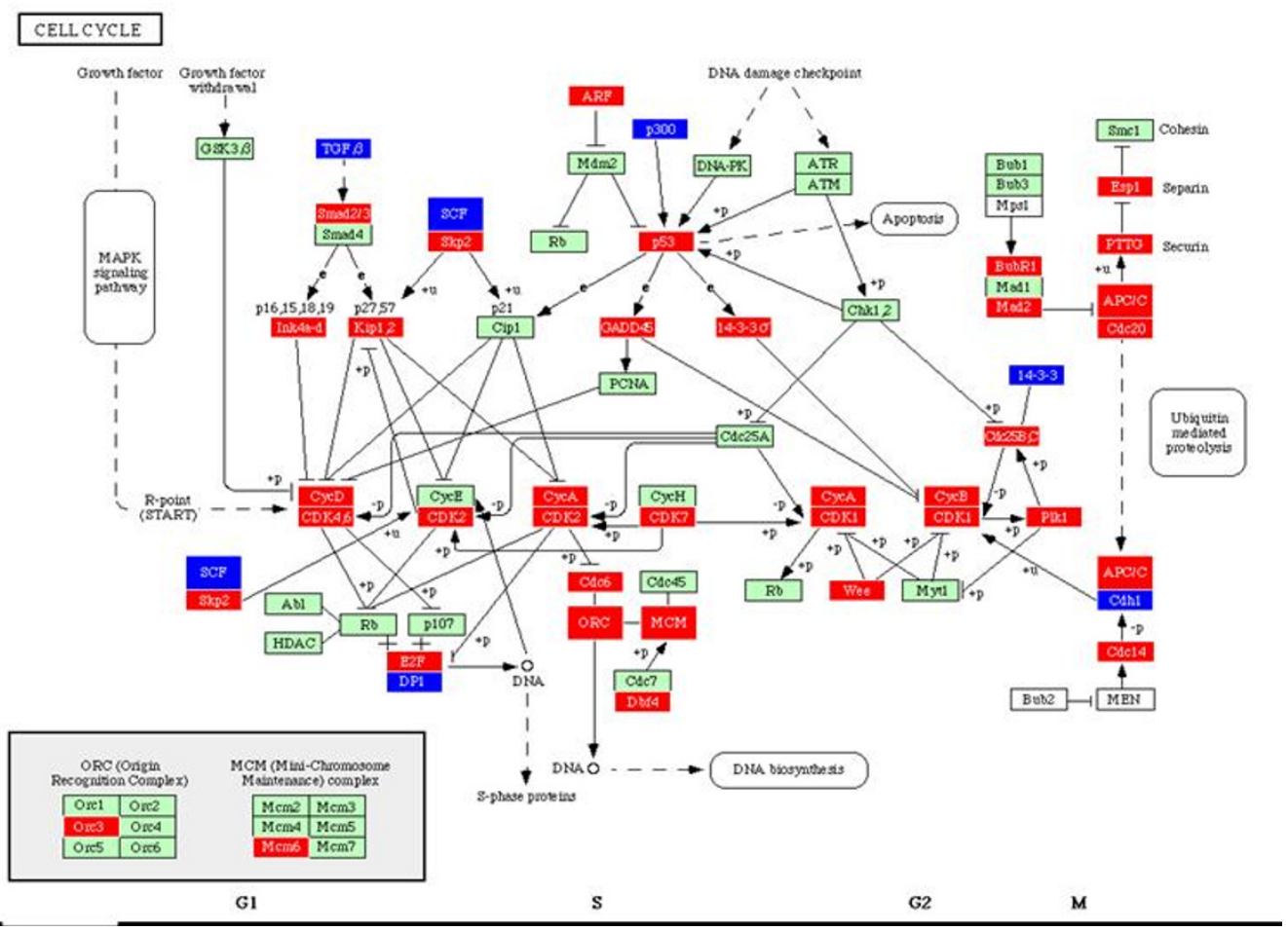

Figure 2

Impact of a mixed microbial challenge on cell cycle control in HIGK. Transcriptional regulation of the KEGG cell cycle pathway modulated by infection with $P$. gingivalis alone $(A)$ or a mixture of $P$. gingivalis and $S$. gordonii $(B)$. Genes represented by red boxes indicate up-regulated transcript levels compared to uninfected controls, where as those genes depicted in blue represent down-regulated transcript levels. 

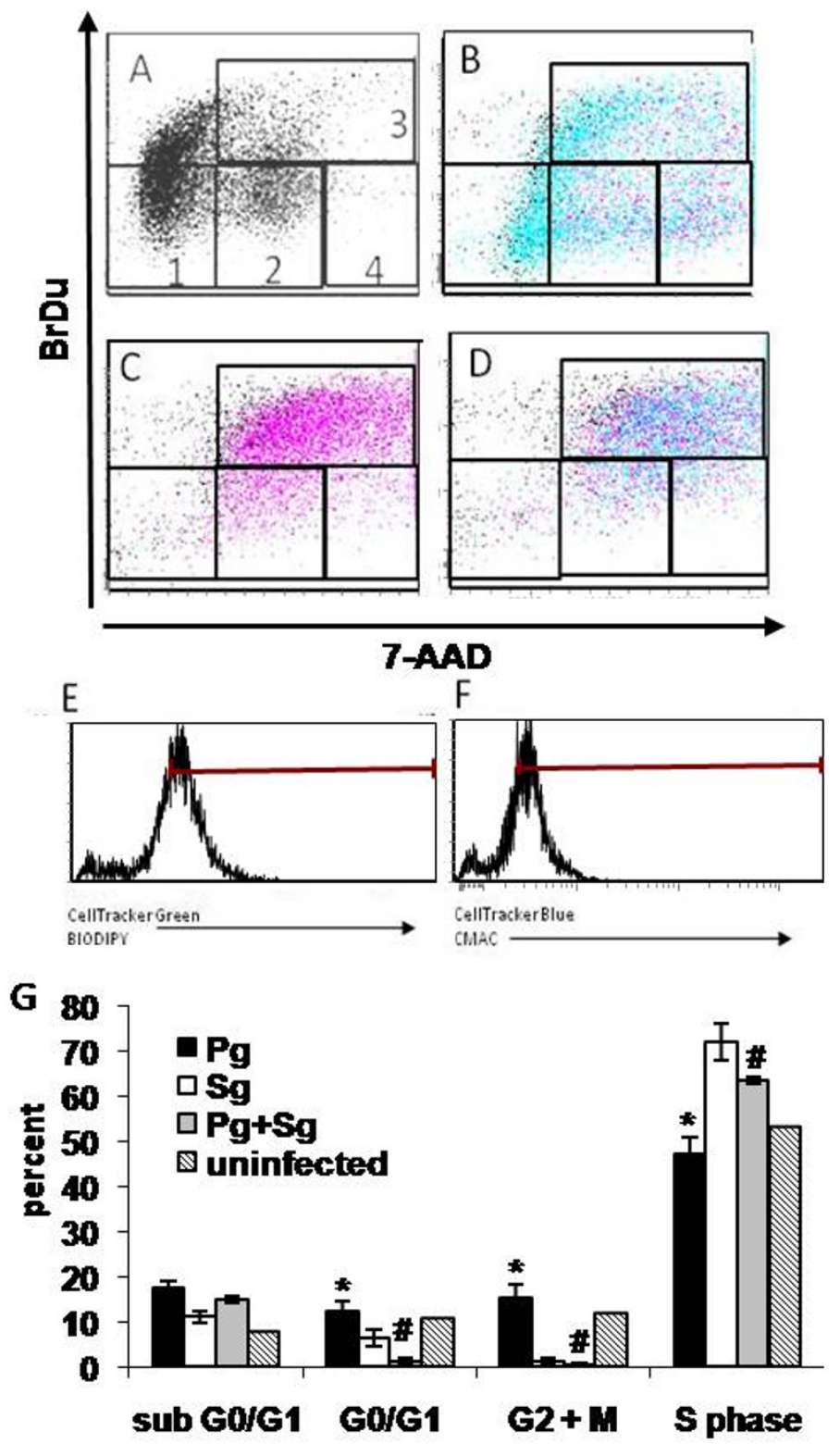

Figure 3

Infection with $\boldsymbol{S}$. gordonii antagonizes the effect of $\boldsymbol{P}$. gingivalis on the cell cycle. HIGK (uninfected in A) were simultaneously grown in the presence of BrdU and infected with $P$. gingivalis labeled with CellTracker Green BIODIPY shown in blue (B), CellTracker Blue CMAC labeled S. gordonii shown in pink (C), or a combination of $P$. gingivalis and S. gordonii for $4 \mathrm{~h}$ (D). HIGK cells were treated with antibiotic to kill extracellular bacteria and were cultured for an additional $20 \mathrm{~h}$ prior to harvesting for FACS analysis. The cell cycle positions and active DNA synthetic activities of cells were determined by analyzing the correlated expression of total DNA (7-AAD) and incorporated BrdU levels. Uninfected control HIGK were included for comparison of baseline cycling patterns. Data represent duplicate experiments. Region gate I, HIGK were apoptotic (defined as sub $\left.\mathrm{G}_{0} / \mathrm{G}_{1}\right)$; region $2, \mathrm{G}_{0} / \mathrm{G}_{1}$; region $3, \mathrm{~S}$ phase; region $4, \mathrm{G}_{2}+\mathrm{M}$. Panel $\mathrm{E}$ and $\mathrm{F}$ demonstrate the gated HIGK populations used throughout all the analyses for CellTracker Green labeled $P$. gingivalis and CellTracker Blue CMAC labeled S. gordonii, respectively. Panel G depicts quantitative analysis of HIGK cell cycling in response to mono- and mixed infections. Results are the mean of three experiments. Statistical analysis was conducted using an ANOVA with Bonferroni's Multiple Comparison Test. * $P g$ vs. Sg sub $G_{0} / G_{1}, P g$ vs. $S g G_{0} / G_{1}, P g$ vs. $S g G_{2}+M, P g$ vs. Sg S phase, $p$ value $<0.05$. \# Pg vs. Sg+Pg sub $G_{0} / G_{1}, G_{0} / G_{1}, G_{2}+M$, $S$ phase, $P$ values $<0.001$. Error bars represent the mean $\pm S D$. 
was not due to indirect effects, such as $\mathrm{pH}$ changes or nutrient depletion of the growth media, but are the result of direct interaction between $S$. gordonii and HIGK cells because conditioned $S$. gordonii media had no effect on HIGK proliferation (see Additional file 1, Additional file 2, and Additional file 3 ).

\section{Discussion}

Previous studies have elucidated the unique transcriptional signatures that are elicited in response to monoinfection with oral pathogens $P$. gingivalis and A. actinomycetemcomitans [41] as well as opportunistic bacteria such as Fusobacterium nucleatum and the commensal species including $S$. gordonii [42]. Transcriptional responses to $P$. gingivalis or A actinomycetemcomitans mono-infections demonstrated organism-specific responses that are drastically different [41]. In contrast, the opportunistic pathogen Fusobacterium nucleatum and S. gordonii elicit profiles that are more transcriptionally restrained in comparison to those characterizing the overt periopathogens [42]. Interestingly, the transcriptional changes that have been observed in HIGK infected with different commensal or pathogenic species does not correlate with the levels of invasiveness that are characteristic of these bacterial species. For example, F. nucleatum invades epithelial cells, while S. gordonii does not. Yet, both species induce very similar transcriptional responses in HIGK [42]. This considerable overlap suggests an evolutionary-driven programmed response to the presence of indigenous constituents of the normal human oral flora. Hence, gingival epithelial cells can differentiate between commensal or potentially pathogenic species, regardless of their cellular localization, and respond accordingly. In this study, the dichotomy in the responses to pathogen or commensal supports previous reports that suggested a species-specific recognition that was tailored to each different bacterial species $[41,42]$.

The microbial challenge faced by the subgingival area is one of great complexity and is dynamic in status. The host epithelial response to in vivo oral biofilms would be extremely difficult to mimic, as an in vitro biofilm model may not exactly duplicate the complex microbiome present in the oral cavity. Furthermore, the strata of organisms present in oral biofilms/plaque vary widely amongst individuals [43]. As such, there are few studies that have investigated bacterial pathogenicity in complex infection models. Nevertheless, the current work offers proof-ofprinciple that an admittedly overly simplified mixed microflora can have profound effects on the host transcriptome, as compared to mono-species bacterial challenges. To date, this is the first characterization of the epithelial response to a mixed infection encompassing commensal and pathogenic organisms. When S. gordonii and $P$. gingivalis were co-cultured together with HIGK, the resulting transcriptional response of the host cell was most similar to that elicited by infection with the S. gordonii alone. This finding supports the hypothesis that commensal species modulate the pathogenicity of $P$. gingivalis in vivo. This result was not surprising considering that microbe-microbe interactions between $P$. gingivalis and $S$. gordonii modulate the gene expression pattern of $P$. gingivalis [26].

In our model, the most significant effect of a mixed challenge was the impact on host cell cycling. HIGK infected with $P$. gingivalis were more evenly distributed in all phases of the cell cycle compared to cells infected with both $P$. gingivalis and $S$. gordonii. HIGK exposed to this mixed co-culture infection, as well as $S$. gordonii alone were more prevalently found in Synthesis phase. Whether this is a true stage arrest phenomenon elicited by the presence of $S$. gordonii could not be determined since cells were not $a$ priori synchronized. Nevertheless, cell growth analysis corroborated the ability of $P$. gingivalis to induce cellular proliferation as compared to uninfected controls. Further, mixed infections with $S$. gordonii were capable of inhibiting the cellular proliferation induced by $P$. gingivalis in a dose-dependant fashion.

Our microarray analyses are in line with previous proteomic experiments performed in primary gingival epithelial cells (GEC) [44]. Both studies found that several pathways exerting regulatory control over the cell cycle were impacted by $P$. gingivalis mono-infection. Both studies also consistently showed a regulation of Cdk2 and Cdk4/6 upon infection of epithelial cells with $P$. gingivalis. Both Cdk2 and Cyclin D were down-regulated in response to infection with $P$. gingivalis with respect to transcript levels and protein levels. Conversely, transcript levels were increased in response to a mixed infection with $S$. gordonii. Only by combining genomic, proteomic, and phenotypic epithelial responses can a true picture emerge of what impact the increasing complexity of a microbiome has on host cellular responses.

Recent evidence supports the concept that cell cycle is significantly impacted in diseased sites as compared to matching healthy sites in periodontitis and gingivitis patients [45]. Although arguably more clinically relevant, studies involving human specimens or primary cell culture present the greatest potential for uncontrolled experimental variables. Examples include the genetic variability between donors, different levels of inflammation and age of participant, diet, diurnal variations in gene expression, type of anesthesia used, length of ischemia prior to tissue removal, time from tissue removal to RNA stabilization, and other confounding factors [12]. In contrast, experiments performed with immortalized cell cultures although simplistic models of the in vivo environment - 


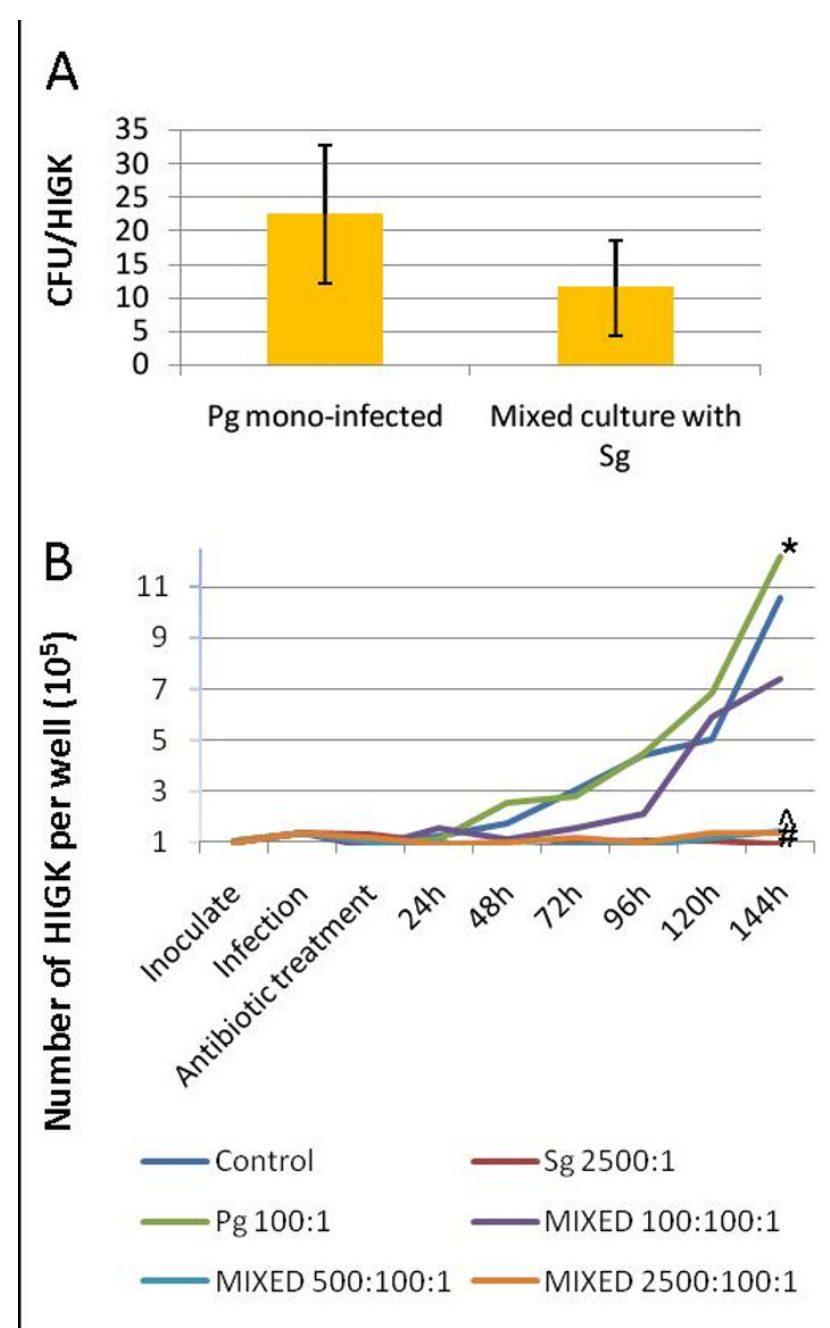

\section{Figure 4}

Accelerated HIGK proliferation upon infection with $\boldsymbol{P}$. gingivalis is inhibited in co-culture with $S$. gordonii. A) Total interaction and invasion levels determined by measuring the total numbers of $P$. gingivalis associated with HIGK cells by live counts in mono- $(\mathrm{Pg})$ and in mixed infection (Mixed) with S. gordonii at the highest MOI. Results are the mean of three experiments. B) HIGK cells at a low confluency were co-cultured with single and complex mixtures of bacteria and cultured for up to I44 hours. Labels: Control, uninfected HIGK; Sg, S. gordonii; Pg, P. gingivalis; MIXED, coinfection with $S$. gordonii and $P$. gingivalis at different multiplicity of infection (MOI). All cell counts were performed in triplicate and all experiments were repeated twice. ${ }^{*} p>0.05 P$. gingivalis vs non-infected controls; \#p $<0.05 \mathrm{~S}$. gordonii vs. non-infected controls; ${ }^{\wedge} p<0.05 \mathrm{~S}$. gordonii co-infection with $P$. gingivalis $(2500: I)$ vs. non-infected controls $(p<0.05)$ by ANOVA with Dunnett's Multiple Comparison Test.

present a significantly higher degree of stability and can be manipulated. This translates into a dataset that is less noisy and ultimately presents a greater potential to dissect a given pathway and predict a phenotype with biological relevance. Hence, the current transcriptional dataset pro- vided valuable insight on the intricate and complex mechanisms that may be responsible for the differential cell cycle effect of $P$. gingivalis and $S$. gordonii, and provided clues on how the presence of the latter in a mixed infection may affect the proliferative properties of $P$. gingivalis. For example, CycD, CDK4 and CDK6, were upregulated in HIGK infected with the mixed microflora compared to HIGK transcript levels in response to mono-infection with P. gingivalis. At the beginning of the cell cycle, Cyclin D and CDK4 and 6 form complexes in response to extracellular signals for growth and stimulate entry into $G_{1}$. The same pattern held for CDK2 which complexes with several different cyclins, but when bound to cyclin E pushes the cell from $G_{1}$ to $S$ phase $\left(G_{1} / S\right.$ transition). Cyclin $A$, which when complexed with CDK2 initiates the $G_{2} / M$ transition, was upregulated in the presence of a mixed infection of HIGK. Transcript levels of all of these cell cycle regulators were down regulated in $P$. gingivalis infected cells compared to uninfected controls. Down regulation of Cyclin A transcript in $P$. gingivalis infected HIGK fits with regulation of the rest of the cell cycle modulating factors because Cyclin A, when complexed with CDK1, also functions as a S-phase promoting factor. Many regulatory steps in protein production, folding, and function as well as posttranscriptional kinase activities can also impact protein function. These post-transcriptional modifications were not detected by transcriptional profiling, yet may significantly alter the initial epithelial transcriptional response with any bacterium. While the aforementioned cyclins and CDKs play a large role in determining the stage of cell cycle progression, ultimately these regulatory molecules control the activity of the transcriptional activator E2F. The activation of E2F results in transcription of a number of genes that promote the cell's transition from $G_{1}$ to $S$ phase via phosphorylation and deactivation of the $R b$ protein. E2F transcript levels were up-regulated in HIGK infected with both $P$. gingivalis and $S$. gordonii and downregulated in cells mono-infected with $P$. gingivalis. CDC6, which is required for the initiation of DNA replication, was similarly regulated in both treatment conditions. Further validation of the current transcriptional dataset is ongoing in primary GECs utilizing clinical biofilm specimens.

Besides the cell cycle pathway, additional pathways were differentially impacted by mono- versus mixed infections, and are currently being further confirmed at the protein level and phenotypically. These pathways include apoptosis and numerous signaling pathways (Table 1). In addition, genes associated with cancer were differentially impacted by infection with $P$. gingivalis, which is not surprising since genes involved in normal physiological functions are often also implicated in cancer when they are disregulated. It remains unclear and speculative whether $P$. gingivalis is directly involved in the initiation or exacerbation of carcinogenic lesions or whether this effect is 
consequent to the attempt of $P$. gingivalis to establish an anti-apoptotic phenotype in GECs. This phenomena has already been shown to help this microorganism to propagate a suitable niche for an extended infection and involves manipulating pathways that are normally involved in normal cellular functions as well as in cancer $[16,41,42,46,47]$. Hence, further endeavors into the possible carcinogenic potential of periodontal pathogens and the dissection of the role of commensal species in affecting these pathways is appropriate and timely.

\section{Conclusion}

In summary, the present study provides evidence that the degree of complexity of a mixed microbiota influences the transcriptional response to infection of host epithelial cells. The transcriptional repertoire of genes impacted by co-infection with $S$. gordonii and $P$. gingivalis compared to that of HIGK infected with $P$. gingivalis alone demonstrated that commensal species are able to modulate expression of host genes with a broad diversity of physiological functions and antagonize the effect of pathogenic species at the cellular level. This global expression study provides insight into both host-pathogen interactions within the context of a complex microbial infection and challenges the current dogma regarding the potential versus the actual pathogenicity of an oral species in the context of a complex biofilm. Although $S$. gordonii is consistently referred to as a commensal, its association with the host is not completely inconsequential as it serves as an early colonizer responsible for the development of potentially pathogenic plaque species. The study of the global transcriptome of epithelial cells to single and complex microbial challenges offers clues towards a better understanding of how bacteria-bacteria interactions and bacteria-host interactions impact the overall host response. Ultimately, this work could lead to identifying host responses that can be used to predict a commensal or pathogenic challenge as well as the overall pathogenic potential of a mixed microbiota. Genes and cell pathways impacted by such infections could serve as diagnostic markers to assess the pathogenicity of individual complex microbial communities across various populations battling different manifestations and stages of periodontal disease.

\section{Methods}

\section{Bacterial strains}

Porphyromonas gingivalis strain ATCC 33277 was cultured anaerobically for $24 \mathrm{~h}$ at $37^{\circ} \mathrm{C}$ in trypticase soy broth supplemented with yeast extract $\left(1 \mathrm{mg} \mathrm{mL}^{-1}\right)$, haemin $(5 \mu \mathrm{g}$ $\left.\mathrm{mL}^{-1}\right)$, and menadiaone $(1 \mu \mathrm{g} \mathrm{mL}-1)$. Streptococcus gordonii strain DL1 was cultured aerobically for $24 \mathrm{~h}$ at $37^{\circ} \mathrm{C}$ in Todd Hewlett Broth supplemented with $0.5 \%$ yeast extract and $0.5 \%$ glucose.

\section{Eukaryotic cell lines}

Human immortalized gingival keratinocytes (HIGK) were originally generated by transfection of primary GEC with E6/E7 from HPV [48]. HIGK were cultured under 5\% $\mathrm{CO}_{2}$ in keratinocyte serum-free medium (K-SFM, Gibco/Invitrogen, Carlsbad, CA) supplemented with $0.05 \mathrm{mM}$ calcium chloride, $200 \mathrm{mM}$ L-glutamine (Gibco/Invitrogen), and $1 \%$ antibiotic/antimycotic (Gibco/Invitrogen).

\section{Microbial-host cell co-culture}

Bacteria were harvested by centrifugation and resuspended in antibiotic-free K-SFM media. HIGK ( $10^{7}$ cells) were washed three times with phosphate-buffered saline (PBS) and incubated with bacteria at an MOI of 100 for $P$. gingivalis and 2500 for $S$. gordonii. After $2 \mathrm{~h}$ at $37^{\circ} \mathrm{C}$, the innoculate was removed and HIGK cells were immediately lysed with Trizol reagent (Invitrogen, Carlsbad, CA) before RNA extraction. Co-cultures were carried out in quadruplicate per infection condition. For microbial-host cell interaction studies, total numbers of $S$. gordonii associated with HIGK - both externally and internally - after $2 \mathrm{~h}$ incubation and washing were determined by Triton $\mathrm{X}$ 100 lysis and plate counts. Invasion levels of S. gordonii and $P$. gingivalis co-cultured with HIGK both individually and together in a mixed infection were measured by antibiotic protection assays as previously described [49]. In mixed co-culture with HIGK and $P$. gingivalis mono-infection, cells were lysed with sterile water as $P$. gingivalis were not viable after exposure to Triton X-100.

\section{RNA isolation, cRNA synthesis and chip hybridization}

Total RNA was extracted, DNAse-treated, purified and quantified according to standard methods (Qiagen and Affymetrix). Briefly, double stranded cDNA was synthesized using $8 \mu \mathrm{g}$ of total cellular template RNA according to standardized protocol (SuperScript double stranded cDNA synthesis kit; Invitrogen, Carlsbad, CA). cRNA was transcribed in vitro, incorporating biotinylated nucleotides via a BioArray high-yield RNA transcript labeling kit (T7) (Enzo Life Sciences, Farmingdale, NY), fragmented, and hybridized onto the Affymetrix human genome U133 Plus 2.0 human microarrays. Each condition was studied in biological quadruplicate and samples were not pooled. The microarrays were hybridized for 16 h at $45^{\circ} \mathrm{C}$, stained with phycoerythrin-conjugated streptavidin and washed according to the Affymetrix protocol (EukGE-WS2v4) using an Affymetrix fluidics station, and scanned with an Affymetrix scanner.

\section{Microarray data analysis}

Microarray data analysis was performed as previously described [41,42]. Briefly, expression filters were applied to remove Affymetrix controls and probe-sets whose signal was undetected across all samples. The signal intensity 
values of the resulting dataset were variance-normalized, mean-centered and ranked by their coefficients of variation. Normalization was performed to give equal weight to all probe-sets in the analysis, regardless of the order of magnitude of the raw signal intensity. To reduce the confounding effect of background signal variation on the analysis, only the half of the dataset demonstrating the most variation across samples was used to perform unsupervised hierarchical cluster analysis using Cluster software [34]. The resulting heat-map and cluster dendrograms were visualized with Treeview software [34] to reveal the extent of characteristic host cell responses to each infection state, defined as identical treatments clustering together. Array results have been deposited in the GEO repository http://www.ncbi.nlm.nih.gov/geo/ index.cgi under accession number GSE12121. Following initial assessment of the host cell response to each condition, supervised class prediction was performed to investigate differences in gene regulation among experimental conditions. For this analysis, the raw signal intensities were log-transformed for all probe-sets that passed the initial expression filters, and were correlated using BRB Array Tools [35]. Diagonal linear discriminant analysis, 1- and 3- nearest neighbors, and nearest centroid prediction methods were used in conjunction with a random variance model for univariate F tests. In each supervised analysis, biological replicates were grouped into classes according to their infection state during co-culture experiments and probe sets significant at the $p<0.05$ level between the class were identified. To test the ability of these significant probe sets to truly distinguish between the classes, leave-one-out-cross-validation (LOOCV) studies were performed for each prediction model. In these LOOCV studies each array was left out in turn and classifiers were derived from the remaining 15 samples. The ability of these classifiers to correctly predict the identity of the left out sample was compared to the expected rate due to chance alone ( $25 \%$ for 4 classes). Using the gene expression classifiers derived from linear discriminant analysis and 1-nearest neighbor methods, the arrays were correctly classified $75 \%$ of the time. Classifiers from 3nearest neighbors and nearest centroid correctly predicted the identity of the left-out array with $69 \%$ accuracy. To assess the significance of the LOOCV results, Monte Carlo simulation with 2,000 random permutations of the dataset was also performed. The significance of the LOOCV results was $p<0.001$ for all prediction methods. Characterized KEGG pathways were populated by the resulting list of probesets significant at the $p<0.05$ level (6066) using Pathway Express, available at http://vor tex.cs.wayne.edu/projects.htm[36-39]. The $p$ values for each pathway were calculated using hypergeometric over representation approach with Bonferroni's Multiple Comparisons Test.

\section{Cell cycling analysis of infected HIGK}

$P$. gingivalis were labeled overnight using live dye CellTracker Green BODIPY (Molecular Probes). S. gordonii were labeled with live dye CellTracker Blue CMAC (Invitrogen) in a similar manner. HIGK were infected with the same MOI used in the microarray experiments in the presence of $1 \mathrm{mM}$ BrdU for four hours prior to treatment with antibiotic/antimycotic and gentamicin $\left(300 \mu \mathrm{g} \mathrm{mL}^{-1}\right)$ to kill extracellular bacteria. Cells were then allowed to grow an additional twenty hours before harvesting for FACS analysis. For quantitative analysis, similar infection conditions were used but only $P$. gingivalis was labeled with CellTracker Green BODIPY.

APC BrdU Flow kits were purchased from BD Pharmingen (San Diego, CA) and used for flow cytometric analysis of cell cycle. Briefly, bromodeoxyuridine (BrdU)-pulsed cells were fixed and permeabilized with BD cytofix/cytoperm buffers, after which DNAse was used to expose incorporated BrdU. APC conjugated anti-BrdU was used to label newly incorporated BrdU while 7-amino-actinomycin D (7-AAD) was used to stain total DNA content. Data was acquired using a FACS Calibur (Becton Dickinson, Mountain View, CA) and analyzed using FCS express software (De Novo). Three independent experiments were performed of each condition in triplicate. Statistical analyses were performed using an ANOVA with Bonferroni's Multiple Comparison test.

\section{HIGK growth analysis}

Approximately $10^{5}$ HIGK (ca. 10\% confluence) were seeded to T75 flasks in K-SFM with supplements. The cells were co-cultured with single and complex mixtures of bacteria at $37^{\circ} \mathrm{C}$ in $5 \% \mathrm{CO}_{2}$ for $2 \mathrm{~h}$ at various MOI and under the conditions described above. After infection, the cells were washed three times with PBS and further cultured for up to 144 hours in K-SFM supplemented with antibiotic/ antimycotic (Gibco/Invitrogen) and gentamicin (300 $\mu \mathrm{g}$ $\mathrm{mL}^{-1}$ ). At each time-point, the cells were dissociated using Accutase (Innovative Cell Technologies, San Diego, CA) following the manufacturer's recommendations and cell counts were determined using a Z1 Coulter Particle Counter (Beckman/Coulter). All cell counts were performed in triplicate and all experiments were repeated twice. ANOVA with Dunnett's Multiple Comparison Test was used to determine statistical significance of each infection condition compared to uninfected controls.

\section{List of Abbreviations}

(7-AAD): 7-amino-actinomycin D; (BrdU): bromodeoxyuridine, (CFU): colony forming units; (CDNA): complementary DNA; (cRNA): complementary RNA; (CDK): cyclin-dependent kinase; (DNA): deoxyribonucleic acid; (FACS): fluorescence-activated cell sorting; $\left(\mathrm{G}_{0}, \mathrm{G}_{1}, \mathrm{G}_{2}\right)$ : 
gap/growth phase; (GEC): gingival epithelial cells; (HIGK): human immortalized gingival keratinocytes; (HPV): human papillomavirus; (IL-8): interleukin 8; (KSFM): keratinocyte serum free media; (LOOCV): leaveone-out cross-validation; (LAP): Localized Aggressive Periodontitis; (M): mitosis; (MOI): multiplicity of infection; (PBS): phosphate-buffered saline; (RNA): ribonucleic acid; (S): synthesis phase.

\section{Authors' contributions}

JJM and KvL contributed equally to this work and should be considered co-first authors. JJM participated in the design of the study, aided the microbial-host cell co-culture studies, aided with all microarray experiments and analysis, performed the supplemental HIGK growth analyses experiments, and helped draft the manuscript. KvL participated in the design of the study, carried out the microbial-host cell co-culture studies, the cell cycling analysis and drafted the manuscript. CD conducted confocal laser fluorescence microscopy experiments (data not shown). SW aided in cell cycling analysis. SMW participated in design of the cell cycling analyses, performed the statistical analysis, and helped to draft the manuscript. HVB helped coordinate the microarray data analysis. RJL participated in the design of the study and helped to draft the manuscript. MH conceived the study, participated in its design and coordination, performed the microarray statistical analysis, conducted the HIGK growth analyses experiments, and helped to draft the manuscript. All authors read and approved the final manuscript.

\section{Additional material}

\section{Additional file 1}

"Antagonism of $\mathrm{P}$. gingivalis-induced HIGK proliferation by S. gordonii is not due to indirect effects upon culture media. "line graph showing HIGK cell growth over time under 6 experimental conditions. Click here for file

[http://www.biomedcentral.com/content/supplementary/14712164-10-380-S1.jpeg]

\section{Additional file 2}

"Antagonism of P. gingivalis-induced HIGK proliferation by S. gordonii is not due to indirect effects upon culture media. " word document describing the experimental conditions used to generate file 1 . Click here for file

[http://www.biomedcentral.com/content/supplementary/14712164-10-380-S2.doc]

\section{Additional file 3}

"Supplementary Materials and Methods. Antagonism of P. gingivalis-induced HIGK proliferation by $\mathrm{S}$. gordonii is not due to indirect effects upon culture media. " word document describing the materials and methods used to generate file 1/supplementary figure.

Click here for file

[http://www.biomedcentral.com/content/supplementary/14712164-10-380-S3.doc]

\section{Acknowledgements}

This work was supported by UFCD Summer Research Fellowship (K.v.L., C.D. and S.W.), and NIH/NIDCR T32 Grant DE07200 (J.J.M.), ROI DEI67I5 (M.H.) and ROI DEIIIII (R.J.L). Microarray supervised analyses were performed using BRB ArrayTools developed by Dr. Richard Simon and BRB-ArrayTools Development Team. The authors would like to thank Steve McClellan from the University of Florida ICBR for assistance with cytometric analysis, and Jennifer VanPuymbrouck for assistance with microbiological assays on invasion and adhesion to HIGK.

\section{References}

I. Backhed F, Ding H, Wang T, Hooper LV, Koh GY, Nagy A, Semenkovich CF, Gordon Jl: The gut microbiota as an environmental factor that regulates fat storage. Proc Natl Acad Sci USA 2004, I0I(44): |57|8-|5723.

2. Hooper LV, Gordon Jl: Commensal host-bacterial relationships in the gut. Science 200I, 292(55 I9): I II5-III8.

3. Krisanaprakornkit S, Kimball JR, Weinberg A, Darveau RP, Bainbridge BW, Dale BA: Inducible expression of human beta-defensin 2 by Fusobacterium nucleatum in oral epithelial cells: multiple signaling pathways and role of commensal bacteria in innate immunity and the epithelial barrier. Infect Immun 2000, 68(5):2907-29I5.

4. Cosseau C, Devine DA, Dullaghan E, Gardy JL, Chikatamarla A, Gellatly S, Yu LL, Pistolic J, Falsafi R, Tagg J, Hancock RE: The commensal Streptococcus salivarius $K 12$ downregulates the innate immune responses of human epithelial cells and promotes host-microbe homeostasis. Infect Immun 2008, 76(9):4I63-4I75.

5. Neish AS, Gewirtz AT, Zeng H, Young AN, Hobert ME, Karmali V, Rao AS, Madara JL: Prokaryotic regulation of epithelial responses by inhibition of IkappaB-alpha ubiquitination. Science 2000, 289(5484): I560-I563.

6. Macpherson AJ, Uhr T: Induction of protective IgA by intestinal dendritic cells carrying commensal bacteria. Science 2004, 303(5664): 1662-1665.

7. Zhang G, Chen R, Rudney JD: Streptococcus cristatus attenuates Fusobacterium nucleatum-induced interleukin-8 expression in oral epithelial cells. J Periodontal Res 2008, 43(4):408-4I6.

8. Socransky SS, Haffajee AD, Ximenez-Fyvie LA, Feres M, Mager D Ecological considerations in the treatment of Actinobacillus actinomycetemcomitans and Porphyromonas gingivalis periodontal infections. Periodontol I999, 20:34|-362.

9. Socransky SS, Haffajee AD, Cugini MA, Smith C, Kent RL Jr: Microbial complexes in subgingival plaque. J Clin Periodontol 1998, 25(2): | $34-\mid 44$

10. Slots J, Genco RJ: Black-pigmented Bacteroides species, Capnocytophaga species, and Actinobacillus actinomycetemcomitans in human periodontal disease: virulence factors in colonization, survival, and tissue destruction. J Dent Res 1984, 63(3):4|2-42|.

II. Slots ]: Bacterial specificity in adult periodontitis. A summary of recent work. J Clin Periodontol I986, I3(10):912-917.

12. Handfield M, Baker HV, Lamont RJ: Beyond Good and Evil in the Oral Cavity: Insights into Host-Microbe Relationships Derived from Transcriptional Profiling of Gingival Cells. Dent Res 2008, 87(3):203-223.

13. Mans JJ, Lamont RJ, Handfield M: Microarray analysis of human epithelial cell responses to bacterial interaction. Infect Disord Drug Targets 2006, 6(3):299-309.

14. Ichikawa JK, Norris A, Bangera MG, Geiss GK, van 't Wout AB, Bumgarner RE, Lory S: Interaction of pseudomonas aeruginosa with epithelial cells: identification of differentially regulated genes by expression microarray analysis of human cDNAs. Proc Nat Acad Sci USA 2000, 97(17):9659-9664.

15. Lory S, Ichikawa JK: Pseudomonas-epithelial cell interactions dissected with DNA microarrays. Chest 2002, I2I(3 Suppl):36S-39S.

16. Mao S, Park Y, Hasegawa Y, Tribble GD, James CE, Handfield M, Stavropoulos MF, Yilmaz O, Lamont RJ: Intrinsic apoptotic pathways of gingival epithelial cells modulated by Porphyromonas gingivalis. Cell Microbiol 2007, 9(8): 1997-2007. 
17. Yilmaz O, Watanabe K, Lamont RJ: Involvement of integrins in fimbriae-mediated binding and invasion by Porphyromonas gingivalis. Cell Microbiol 2002, 4(5):305-3I4.

18. Rudney JD, Chen R, Sedgewick G]: Actinobacillus actinomycetemcomitans, Porphyromonas gingivalis, and Tannerella forsythensis are components of a polymicrobial intracellular flora within human buccal cells. J Dent Res 2005, 84(I):59-63.

19. Rudney JD, Chen R, Sedgewick GJ: Intracellular Actinobacillus actinomycetemcomitans and Porphyromonas gingivalis in buccal epithelial cells collected from human subjects. Infect Immun 200I, 69(4):2700-2707.

20. Lamont RJ, Yilmaz O: In or out: the invasiveness of oral bacteria. Periodontol 2002, 30:61-69.

21. Ellen RP, Lepine G, Nghiem PM: In vitro models that support adhesion specificity in biofilms of oral bacteria. Adv Dent Res I997, I I(I):33-42.

22. Rosan B, Lamont RJ: Dental plaque formation. Microbes Infect 2000, 2(13):1599-1607.

23. Yao ES, Lamont RJ, Leu SP, Weinberg A: Interbacterial binding among strains of pathogenic and commensal oral bacterial species. Oral Microbiol Immunol I996, I I(I):35-4I.

24. Lamont RJ, El-Sabaeny A, Park Y, Cook GS, Costerton JW, Demuth DR: Role of the Streptococcus gordonii SspB protein in the development of Porphyromonas gingivalis biofilms on streptococcal substrates. Microbiology 2002, I 48(Pt 6): 1627-1636.

25. Xie H, Cook GS, Costerton JW, Bruce G, Rose TM, Lamont RJ: Intergeneric communication in dental plaque biofilms. J Bacteriol 2000, I82(24):7067-7069.

26. Simionato MR, Tucker CM, Kuboniwa M, Lamont G, Demuth DR, Tribble GD, Lamont RJ: Porphyromonas gingivalis genes involved in community development with Streptococcus gordonii. Infect Immun 2006, 74(I I):64I9-6428.

27. Maeda K, Nagata H, Yamamoto $Y$, Tanaka M, Tanaka J, Minamino N Shizukuishi S: Glyceraldehyde-3-phosphate dehydrogenase of Streptococcus oralis functions as a coadhesin for Porphyromonas gingivalis major fimbriae. Infect Immun 2004, 72(3): $134 \mid-1348$.

28. Park Y, Simionato MR, Sekiya K, Murakami Y, James D, Chen W, Hackett M, Yoshimura F, Demuth DR, Lamont RJ: Short fimbriae of Porphyromonas gingivalis and their role in coadhesion with Streptococcus gordonii. Infect Immun 2005, 73(7):3983-3989.

29. Demuth DR, Irvine DC, Costerton JW, Cook GS, Lamont RJ: Discrete protein determinant directs the species-specific adherence of Porphyromonas gingivalis to oral streptococci. Infect Immun 200I, 69(9):5736-574I.

30. Kolenbrander PE, Palmer RJ Jr, Rickard AH, Jakubovics NS, Chalmers $\mathrm{NI}$, Diaz PI: Bacterial interactions and successions during plaque development. Periodontol 2006, 42:47-79.

31. Ximenez-Fyvie LA, Haffajee AD, Socransky SS: Microbial composition of supra- and subgingival plaque in subjects with adult periodontitis. J Clin Periodontol 2000, 27( ( 10):722-732.

32. Lamont RJ, Jenkinson HF: Life below the gum line: pathogenic mechanisms of Porphyromonas gingivalis. Microbiol Mol Biol $\operatorname{Rev} 1$ 1998, 62(4): I 244-1263

33. Tribble GD, Mao S, James CE, Lamont RJ: A Porphyromonas gingivalis haloacid dehalogenase family phosphatase interacts with human phosphoproteins and is important for invasion. Proc Natl Acad Sci USA 2006, I03(29): I I 027-I I 032

34. Eisen MB, Spellman PT, Brown PO, Botstein D: Cluster analysis and display of genome-wide expression patterns. Proc Nat Acad Sci USA 1998, 95(25): | 4863-| 4868.

35. Wright GW, Simon RM: A random variance model for detection of differential gene expression in small microarray experiments. Bioinformatics 2003, 19(18):2448-2455.

36. Draghici S, Khatri P, Bhavsar P, Shah A, Krawetz SA, Tainsky MA: Onto-Tools, the toolkit of the modern biologist: OntoExpress, Onto-Compare, Onto-Design and Onto-Translate. Nucleic Acids Res 2003, 3 I ( I3):3775-378I.

37. Khatri P, Bhavsar P, Bawa G, Draghici S: Onto-Tools: an ensemble of web-accessible, ontology-based tools for the functional design and interpretation of high-throughput gene expression experiments. Nucleic Acids Res 2004:W449-56.

38. Khatri P, Sellamuthu S, Malhotra P, Amin K, Done A, Draghici S: Recent additions and improvements to the Onto-Tools. Nucleic Acids Res 2005:W762-5.
39. Khatri P, Voichita C, Kattan K, Ansari N, Khatri A, Georgescu C, Tarca AL, Draghici S: Onto-Tools: new additions and improvements in 2006. Nucleic Acids Res 2007:W206-II.

40. Bloom J, Cross FR: Multiple levels of cyclin specificity in cellcycle control. Nat Rev Mol Cell Biol 2007, 8(2): 149-160.

4I. Handfield M, Mans J], Zheng G, Lopez MC, Mao S, Progulske-Fox A Narasimhan G, Baker HV, Lamont RJ: Distinct transcriptional profiles characterize oral epithelium-microbiota interactions. Cell Microbiol 2005, 7(6):8I I-823.

42. Hasegawa Y, Mans JJ, Mao S, Lopez MC, Baker HV, Handfield M, Lamont RJ: Gingival epithelial cell transcriptional responses to commensal and opportunistic oral microbial species. Infect Immun 2007, 75(5):2540-2547.

43. Paster BJ, Boches SK, Galvin JL, Ericson RE, Lau CN, Levanos VA, Sahasrabudhe A, Dewhirst FE: Bacterial diversity in human subgingival plaque. J Bacteriol 200I, I 83(I 2):3770-3783.

44. Kuboniwa M, Hasegawa Y, Mao S, Shizukuishi S, Amano A, Lamont RJ, Yilmaz O: $P$. gingivalis accelerates gingival epithelial cell progression through the cell cycle. Microb Infect 2007, I0(2): I22- 128.

45. Demmer RT, Behle JH, Wolf DL, Handfield M, Kebschull M, Celenti R, Pavlidis P, Papapanou PN: Transcriptomes in Healthy and Diseased Gingival Tissues. J Periodontol 2008, 79(II):2II 2-2I 24

46. Nakhjiri SF, Park Y, Yilmaz O, Chung WO, Watanabe K, El-Sabaeny A, Park K, Lamont RJ: Inhibition of epithelial cell apoptosis by Porphyromonas gingivalis. FEMS Microbiol Lett 200I, 200(2): I45-I49.

47. Yilmaz O, Jungas $T$, Verbeke $P$, Ojcius DM: Activation of the phosphatidylinositol 3-kinase/Akt pathway contributes to survival of primary epithelial cells infected with the periodontal pathogen Porphyromonas gingivalis. Infect Immun 2004, 72(7):3743-375I.

48. Oda D, Bigler L, Lee P, Blanton R: HPV immortalization of human oral epithelial cells: a model for carcinogenesis. Exp Cell Res 1996, 226(I): 164-169.

49. Lamont RJ, Chan A, Belton CM, Izutsu KT, Vasel D, Weinberg A: Porphyromonas gingivalis invasion of gingival epithelial cells. Infect Immun 1995, 63(10):3878-3885.
Publish with BioMed Central and every scientist can read your work free of charge

"BioMed Central will be the most significant development for disseminating the results of biomedical research in our lifetime. "

Sir Paul Nurse, Cancer Research UK

Your research papers will be:

- available free of charge to the entire biomedical community

- peer reviewed and published immediately upon acceptance

- cited in PubMed and archived on PubMed Central

- yours - you keep the copyright
BioMedcentral 